NORDITA-2011-24

\title{
Dirac equation for membranes
}

\author{
M. Trzetrzelewski, $*$
}

(Dated: July 11, 2021)

\begin{abstract}
Dirac's idea of taking the square root of constraints is applied to the case of extended objects concentrating on membranes in $D=4$ space-time dimensions. The resulting equation is Lorentz invariant and predicts an infinite hierarchy of positive and negative masses (tension). There are no tachyonic solutions.
\end{abstract}

\section{MOTIVATION}

In the Dirac membrane model 1 the hamiltonian corresponding to the spherically symmetric solution includes a square root of the anharmonic oscillator. As noted by Dirac to introduce the spin into the theory one would have to get rid of the square root by bringing in the spin matrices. However, in the vast literature on membranes (see e.g. 2] and references therein) a different way of introducing fermions for extended objects is usually considered by writing the supersymmetric version of the bosonic action 3. Besides aesthetic reasons this seems quite not in the direction indicated by Dirac. It is perhaps worth mentioning that for the case of point particles the Klein-Gordon equation has its supersymmetric counterpart (i.e. the massive Wess-Zumino model) which however does not describe the electron unlike the square root of the Klein-Gordon equation.

In this paper we choose a route indicated by Dirac i.e. introduce fermion fields (and hence spin) for membranes by performing a certain square root. We find it more appropriate to do so for the constraints of the theory rather then for the hamiltonian since the former correspond to the mass shell constraint for point-like particles and are written in relativistically invariant way. However, taking the square root of the hamiltonian is also possible and was discussed in 4 .

The model presented here is manifestly Lorentz invariant hence there are no anomalies originating form special relativity. There are also no tachyonic solutions - we show that in the case of a spherical membrane the spectrum is real and discrete. The eigenvalues are identified with the membrane masses and can be positive as well as negative corresponding to positive respectively negative membrane tension. The field content (and their equations) considered here is novel: the membrane is described by a fermionic field governed by the analog of the Dirac equation. It is an alternative way of describing extended objects in space-time.

Lastly we generalize the discussion by including a coupling to a three form as well as suggest an alternative way of writing the Dirac equation for membranes.

\section{A SQUARE ROOT}

A Lorentz invariant action describing membranes is given by [1]

$$
S_{\text {membrane }}=-\Lambda \int \sqrt{G} d \sigma^{3}, \quad G_{\alpha \beta}:=\partial_{\alpha} X_{\mu} \partial_{\beta} X^{\mu}
$$

where $\Lambda$ is the tension (here we assume $\Lambda>0$ and will comment on $\Lambda<0$ later $), \sigma^{\alpha}=\left(\tau, \sigma^{r}\right)$ is the internal parametrization of the membrane, $X^{\mu}$ are the embedding variables and $G$ is the determinant of the induced metric $G_{\alpha \beta}$. The momenta $\mathcal{P}_{\mu}:=\partial \mathcal{L} / \partial \dot{X}^{\mu}$ satisfy the constraints (see e.g. [5])

$$
\mathcal{P}_{\mu} \mathcal{P}^{\mu}=\Lambda^{2} \operatorname{det} G_{r s}
$$

and follow from the diffeomorphism invariance of (1). In addition to (2) we also have $\mathcal{P}^{\mu} \partial_{r} X_{\mu}=0$.

Equation (2) is a counterpart of the mass shell constraint for a point-like particle therefore in order to keep the analogy with the way in which Dirac obtained equation for fermions it is desirable to perform the square root of (2).

\section{Spherically symmetric membrane}

Let us now consider a concrete example of a sphere at rest

$$
\begin{gathered}
X^{0}=\tau, \quad X^{1}=r(\tau) \sin \varphi \cos \theta, \quad X^{2}=r(\tau) \sin \varphi \sin \theta, \\
X^{3}=r(\tau) \cos \varphi, \quad \varphi \in[0, \pi], \quad \theta \in[0,2 \pi) .
\end{gathered}
$$

The determinant in 22 is $\operatorname{det} G_{r s}=r^{4} \sin ^{2} \varphi$ hence the corresponding square root is

$$
\gamma^{\mu} \mathcal{P}_{\mu}=-\Lambda A r^{2} \sin \varphi
$$


where $A$ is a matrix s.t. $A^{2}=\mathbf{1}, \gamma^{\mu}$ are gamma matrices, $\left\{\gamma^{\mu}, \gamma^{\nu}\right\}=2 \eta^{\mu \nu} \mathbf{1}$, the minus sign is a convention.

Equation (3) is $\varphi$ dependent. A way to obtain the analog of the Dirac equation for the radial variable is to average (3) over the membrane surface by introducing the canonical momenta $\pi_{\mu}$ for the whole membrane via

$$
\pi_{\mu}:=\int_{\tau=\text { const }} \mathcal{P}_{\mu} d \varphi d \theta
$$

This implies

$$
\gamma^{\mu} \pi_{\mu}=-M, \quad M:=\Lambda r^{2} \int A \sin \varphi d \varphi d \theta
$$

and suggests to consider the Dirac equation (substituting $\left.\pi_{\mu}=-i \partial_{\mu}\right)$ of the form

$$
\left(-i \gamma^{\mu} \partial_{\mu}+M\right) \psi=0
$$

where $\psi=\psi(r)$.

Because the spinor $\psi$ is independent of $\theta$ and $\varphi$ we may consider only the radial part of the differential operator in (4). The matrix $A$ should be chosen such that $M$ is proportional to the unit matrix. For $A=\mathbf{1}$ (other choices of $A$ are possible which after averaging lead to $M=0$ ), going to the radial equation (we use the conventions of [6]) we obtain

$$
\left(\partial_{x}+\frac{\kappa}{x}\right) G=\left(\epsilon+x^{2}\right) F, \quad\left(-\partial_{x}+\frac{\kappa}{x}\right) F=\left(\epsilon-x^{2}\right) G
$$

where $x=r(4 \pi \Lambda)^{1 / 3}$ and $\epsilon=E /(4 \pi \Lambda)^{1 / 3}$ are dimensionless, $E$ is the energy, $\kappa= \pm 1$ and $F(x), G(x)$ are the radial parts of the spinor $\psi[6]$. Using matrix notation (5) can be written as

$$
H \phi=\epsilon \phi, \quad H:=\left(\begin{array}{cc}
-x^{2} & \partial_{x}+\frac{\kappa}{x} \\
-\partial_{x}+\frac{\kappa}{x} & x^{2}
\end{array}\right), \quad \phi:=\left(\begin{array}{c}
F \\
G
\end{array}\right) .
$$

The spectrum of $H$ is independent of the choice of $\kappa$ (i.e. if $\phi^{T}=(F, G)$ solves $H \phi=\epsilon \phi$ for $\kappa=1$ then $\phi^{T}=(G, F)$ solves $H \phi=\epsilon \phi$ for $\left.\kappa=-1\right)$ hence we choose $\kappa=1$ from now on. Let us note that if $\phi$ is the eigenvector of $H$ then (due to the $1 / x$ term) $F(0)=$ $G(0)=0$. Therefore we may consider the Hilbert space of square-integrable vectors $\phi$ on $[0, \infty)$ satisfying $\phi(0)=0$. In such Hilbert space the operator $\partial_{x}$ is antihermitian hence $H$ is hermitian which proves that the spectrum of $H$ is real.

Let us also observe that $H^{2}$ can be written as

$$
H^{2}=Q^{2}+\left(\begin{array}{cc}
0 & 0 \\
0 & 2 / x^{2}
\end{array}\right), \quad Q:=\left(\begin{array}{cc}
-x^{2} & \partial_{x} \\
-\partial_{x} & x^{2}
\end{array}\right)
$$

hence the spectrum of $H^{2}$ is discrete, due to the inequality $H^{2} \geq Q^{2}$ and the fact that $Q^{2}$ is discrete (to see explicitly that $Q^{2}$ is discrete it is useful to introduce $a_{ \pm}:=F \pm G$ for which the eigen-equation $Q^{2} \phi=\eta^{2} \phi$ gives $h_{ \pm} a_{ \pm}=\eta^{2} a_{ \pm}, \quad h_{ \pm}:=-\partial_{x}^{2}+x^{4} \pm 2 x$, i.e. $a_{+}$and $a_{-}$decouple and since $h_{+}$and $h_{-}$are discrete, $Q^{2}$ must also be discrete). This proves that the spectrum of $H^{2}$ and hence $H$ is discrete.

To find the exact spectrum of $H$ we use numerical methods (see the Appendix for details). The first positive energy levels are

$$
\epsilon \approx 2.7,4.0,5.1,6.1,7.0,7.9,8.7,9.5, \ldots
$$

while the negative ones are

$$
-\epsilon \approx 1.7,3.3,4.5,5.6,6.6,7.5,8.3,9.1, \ldots
$$

The four degrees of freedom of the spinor $\psi$ should be interpreted just like in the Dirac equation i.e. particles and antiparticles with spin (the notion of spin is not related here to any geometrical configuration such as e.g. the spinning membrane). The antiparticle corresponds to the negative energy $-E$ however the energy in this membrane model is a mass. Therefore one concludes that the model predicts the existence of particles with negative masses. Because the energy is measured in units of $\Lambda^{1 / 3}$ and since $(-\Lambda)^{1 / 3}=-\Lambda^{1 / 3}$ we can identify the negative masses with the negative tension - a possibility already considered by Dirac (see the second reference in [1]). Such objects are unstable (unless one considers gravitational effects with extra volume term in the action [1]). The inclusion of the electromagnetic field would only worsen the stability of particles with negative tension.

The electromagnetic field can be added using minimal coupling prescription $\mathcal{P}_{\mu} \rightarrow \mathcal{P}_{\mu}+e A_{\mu}$ with $A_{0}=-e / 2 r$ (the electrostatic energy of the Coulomb field of a charged sphere) and $A_{i}=0$. Equations (5) stay the same with shifted energy $\epsilon \rightarrow \epsilon+\frac{\alpha}{2 x}$ where $\alpha$ is the fine structure constant. The corresponding hamiltonian is

$$
H^{E M}=H+\frac{\alpha}{2 x} \mathbf{1}
$$

Due to the small value of $\alpha$ the spectrum of $H^{E M}$ is very close to the spectrum of $H$ (differences are of order $10^{-3}$, the lowest positive energy is now 2.7570 compared to 2.7525 obtained previously).

Let us compare the spectrum of $H^{E M}$ with the spectrum of the hamiltonian for the bosonic membrane obtained by Dirac [1]

$$
H_{\text {Dirac }}=\sqrt{-\partial_{r}^{2}+\omega^{2} r^{4}}+\frac{e^{2}}{2 r},
$$

where $\omega=4 \pi \Lambda$. Using the Bohr-Sommerfeld quantization method, Dirac found that the first excitation of the membrane corresponds to the energy $\approx 53 m_{e}$, where $m_{e}$ is the mass of the electron. The exact value turns out 
to be about $43 m_{e}$ as observed in [7. Using the relation $\omega=16 m_{e}^{3} / 27 \alpha^{2}$ [1] we find that our model gives

$$
E=(4 \pi \Lambda)^{1 / 3} \epsilon_{0} \approx \frac{1}{3}\left(\frac{4}{\alpha}\right)^{2 / 3} m_{e} \cdot 2.757=61.5 m_{e}
$$

Therefore we obtained a larger value - in accordance with Dirac's expectation that bringing spin into the theory might lift the eigenvalue. However the result is still not of order of the muon mass.

\section{General case}

Taking the square root of $\sqrt{2}$ is more difficult. While the l.h.s. would simply give $\gamma^{\mu} \mathcal{P}_{\mu}$ the r.h.s. involves a square root of the determinant $\operatorname{det} G_{r s}$ which is 4 th order in derivatives. It turns out that the answer is simple in terms of a hermitian mass matrix density

$$
\mathcal{M}:=\frac{1}{2} i \Lambda \epsilon^{r s} \gamma_{\mu \nu} \partial_{r} X^{\mu} \partial_{s} X^{\nu}
$$

(where $\gamma_{\mu \nu}=\gamma^{\rho \sigma} \eta_{\rho \mu} \eta_{\sigma \nu}, \gamma^{\mu \nu}:=\frac{1}{2}\left[\gamma^{\mu}, \gamma^{\nu}\right], \epsilon^{r s}$ is completely antisymmetric with $\epsilon^{12}=1$ ) due to the identity

$$
\mathcal{M}^{2}=\Lambda^{2} \operatorname{det} G_{r s} \mathbf{1}
$$

which allows to write the square root of the constraint as

$$
\gamma^{\mu} \mathcal{P}_{\mu}=-\mathcal{M}
$$

It is possible to introduce a matrix $\mathcal{A}$ such that the rescaling $\mathcal{M}_{\mathcal{A}}:=\mathcal{A} \cdot \mathcal{M}$ is consistent with the constraint (2). One simple example is $\mathcal{A}=A \mathcal{M} / \Lambda \sqrt{\operatorname{det} G_{r s}}$ where matrix $A$ as s.t. $A^{2}=\mathbf{1}$, for which $\mathcal{M}_{\mathcal{A}}=\Lambda A \sqrt{\operatorname{det} G_{r s}} \mathbf{1}$. Therefore this particular choice of $\mathcal{A}$ will reproduce the results of previous section.

The choice $\mathcal{A}=\mathbf{1}$ results in the averaged mass matrix $M$ equal 0 for spherical membrane, hence the spectrum of the corresponding Dirac operator would be continuous. We now elaborate on this case in more details since it is where quantization procedure is possible in terms of matrix regularization [8].

If the parameters $\sigma^{r}$ are not integrated out the coordinates $X^{\mu}$ are fields and the differential equation of the quantum counterpart of $(9)$ is a functional one. Because $\mathcal{P}_{\mu}$ and $X^{\mu}$ are conjugate variables it is natural to substitute $\mathcal{P}_{\mu}$ with a functional derivative $-i \frac{\delta}{\delta X^{\mu}}$ in (9). A more problematic term is the mass matrix density $\mathcal{M}$. Observing the appearance of the Poisson bracket $\left\{X^{\mu}, X^{\nu}\right\}=\epsilon^{r s} \partial_{r} X^{\mu} \partial_{s} X^{\nu}$ in (7) we write

$$
\left(-i \gamma^{\mu} \frac{\delta}{\delta X^{\mu}}+\frac{1}{2} i \Lambda \gamma_{\mu \nu}\left\{X^{\mu}, X^{\nu}\right\}\right) \Psi=0
$$

which will be useful when using the matrix regularization. The above equation is similar to Eq. (47) of Ref. [9] where an attempt to quantize the bosonic membrane in a covariant fashion was undertaken.

Equation (10) at least formally is solved by

$$
\Psi=e^{S} \Psi_{0}, \quad S=\frac{\Lambda}{12} \gamma_{\mu \nu \rho} \int X^{\mu}\left\{X^{\nu}, X^{\rho}\right\} d^{3} \sigma
$$

where $\Psi_{0}$ is a constant spinor, $\gamma_{\mu \nu \rho}=\frac{1}{3}\left(\gamma_{\mu} \gamma_{\nu \rho}+\right.$ cycl. $)$ and where we used $\gamma^{\mu} \gamma_{\mu \nu \rho}=2 \gamma_{\nu \rho}$. Similar functional expressions were found for the bosonic membrane by Smolin [9], Moncrief [10] and Hoppe [11]. Using the identities $\gamma_{\mu \nu \rho}^{\dagger}=-\gamma_{0} \gamma_{\mu \nu \rho} \gamma_{0}$ and $e^{S^{\dagger}}=\gamma_{0} e^{-S} \gamma_{0}$ it follows that $\bar{\Psi} \Psi=\bar{\Psi}_{0} \Psi_{0}$ therefore $\Psi$ suffers from being non-normalizable (i.e. the functional integral $\int[d X] \bar{\Psi} \Psi$ is infinite).

The hamiltonian formulation can be obtained by singling out the hamiltonian operator $\mathcal{H}:=\mathcal{P}_{0}$ and noting that the gauge choice $X^{0}=\sigma^{0}$ for the coordinates implies that $\left\{X_{0}, X_{\nu}\right\}=0$ hence

$$
\mathcal{H} \Psi=E \Psi, \quad \mathcal{H}=\gamma^{0}\left(\gamma^{i} \mathcal{P}_{i}+\frac{1}{2} \Lambda \gamma_{i j}\left\{X^{i}, X^{j}\right\}\right)
$$

In addition to 10 we also have the constraint $\mathcal{P}_{\mu} \partial_{r} X^{\mu}=$ 0 which after setting the gauge $X^{0}=\sigma^{0}$ becomes $\mathcal{P}_{i} \partial_{r} X^{i}=0$. Locally (on the membrane surface) this constraint is equivalent to $\left\{\mathcal{P}_{i}, X^{i}\right\}=0$ which will be further used. One can write a solution analogous to 11 corresponding to $E=0$

$$
\Psi=e^{S} \Psi_{0}, \quad S=\frac{\Lambda}{6} \gamma_{i j k} \int X^{i}\left\{X^{j}, X^{k}\right\} d^{2} \sigma
$$

(where we used $\gamma^{i} \gamma_{i j k}=\gamma_{j k}$ ) which however is not normalizable by similar argument as before.

Using matrix regularization 8 we can view 12 as the $N \rightarrow \infty$ limit of

$$
H \Psi=E \Psi, \quad H=\gamma^{0} \gamma^{i} p_{i}+\frac{1}{2} \Lambda \gamma^{0} \gamma_{i j}\left[x^{i}, x^{j}\right]
$$

where $p_{i}$ and $x^{i}$ are $s u(N)$ matrices, subject to the constraint $\left[p_{i}, x^{i}\right] \Psi=0$. The solution corresponding to $E=0$ is

$$
\Psi=e^{S} \Psi_{0}, \quad S=\frac{\Lambda}{6 \sqrt{N^{2}-1}} \gamma_{i j k} \operatorname{Tr}\left(x^{i}\left[x^{j}, x^{k}\right]\right)
$$

(using the conventions of [12]). The results of the previous section suggest that the spectrum of membrane excitations does not contain the state with $E=0$ therefore one should not worry that the wavefunctions 13 and (14) are not normalizable. However the exponential factor $e^{S}$ will probably play an important role in search for normalizable excitations. In the matrix-regularized case 
this would imply that the tension has to be renormalized as $\Lambda \sim N$.

The electromagnetic field $A_{\mu}$ can be introduced using the minimal coupling prescription $-i \frac{\delta}{\delta X^{\mu}} \rightarrow-i \frac{\delta}{\delta X^{\mu}}+$ $e A_{\mu}$. How one can write the matrix regularized case is less obvious since $A_{\mu}$ are in general non polynomial.

\section{3-FORM COUPLING}

Let us consider a different approach based on $p$-form electrodynamics 13. which is more natural for extended objects with $p-1$ spatial directions. We therefore assume the existence of a three form $\mathcal{A}$ in space-time, with components given by a completely antisymmetric tensor $\mathcal{A}_{\mu \nu \rho}$ and the corresponding field strength $\mathcal{F}_{\mu \nu \rho \sigma}=\partial_{[\mu} \mathcal{A}_{\nu \rho \sigma]}$

For the action of the membrane coupled with $\mathcal{A}$ we take $S=-\Lambda \int \sqrt{G} d^{3} \sigma-\frac{e}{3 !} \int \epsilon^{\alpha \beta \gamma} \mathcal{A}_{\mu \nu \rho} \partial_{\alpha} X^{\mu} \partial_{\beta} X^{\nu} \partial_{\gamma} X^{\rho} d^{3} \sigma$

so that the momenta and the equations of motion are

$$
\begin{gathered}
\mathcal{P}_{\mu}=-\Lambda \sqrt{|G|} G^{\tau \beta} \partial_{\beta} X_{\mu}-\frac{1}{2} e \mathcal{A}_{\mu \nu \rho}\left\{X^{\nu}, X^{\rho}\right\}, \\
\Lambda \partial_{\alpha} \sqrt{G} G^{\alpha \beta} \partial_{\beta} X^{\mu}+e \mathcal{F}^{\mu}{ }_{\nu \rho \sigma}\left\{X^{\nu}, X^{\rho}, X^{\sigma}\right\}=0
\end{gathered}
$$

where $\left\{X^{\mu}, X^{\nu}, X^{\rho}\right\}:=\epsilon^{\alpha \beta \gamma} \partial_{\alpha} X^{\mu} \partial_{\beta} X^{\nu} \partial_{\gamma} X^{\rho}$ is the Nambu bracket [14]. The corresponding square root of constraints will result in 10 with the functional derivative $-i \frac{\delta}{\delta X^{\mu}} \rightarrow-i \frac{\delta}{\delta X^{\mu}}+\frac{1}{2} e A_{\mu \nu \rho}\left\{X^{\nu}, X^{\rho}\right\}$. One can also write the matrix regularization.

Let us note that the equations of motion for $\mathcal{A}_{\mu \nu \rho}$ do not contradict sourceless Maxwell equations in the following sense: using the identity $\mathcal{F}_{\mu \nu \rho \sigma}=-6 \epsilon_{\mu \nu \rho \sigma} \partial_{\pi} a^{\pi}$ where $a^{\pi}:=\frac{1}{6} \epsilon^{\pi \mu \nu \rho} \mathcal{A}_{\mu \nu \rho}\left(\epsilon_{\mu \nu \rho \sigma}\right.$ is completely antisymmetric, $\epsilon_{1234}=1$ ), the action for $\mathcal{F}$ becomes

$$
S_{\mathcal{F}}=-\frac{1}{2 \cdot 4 !} \int \mathcal{F}_{\mu \nu \rho \sigma} \mathcal{F}^{\mu \nu \rho \sigma} d^{4} x=18 \int\left(\partial_{\mu} a^{\mu}\right)^{2} d^{4} x
$$

i.e. a square of the Lorenz gauge condition. The equations of motion $\partial_{\nu}\left(\partial_{\mu} a^{\mu}\right)=0$ imply $\partial_{\nu} a^{\nu}=$ const. hence the corresponding space of solutions is larger then that of the electromagnetic potential $A_{\mu}$ in the Lorenz gauge. Moreover the residual gauge invariance of $\mathcal{F}^{2}$ is present with $a_{\mu} \rightarrow a_{\mu}+\partial_{\mu} \chi, \square \chi=0$ just like in the formulation of electrodynamics in the Lorenz gauge.

\section{ALTERNATIVE SQUARE ROOT}

An identity similar to (8) holds also for the whole world-volume metric i.e.

$$
\mathcal{L}^{2}=\operatorname{det} G_{\alpha \beta} \mathbf{1}, \quad \mathcal{L}:=\frac{i}{3 !} \gamma_{\mu \nu \rho}\left\{X^{\mu}, X^{\nu}, X^{\rho}\right\} .
$$

Both identities have also a heuristic origin when introducing a matrix line element $d X:=\gamma_{\mu} d x^{\mu}$ and noting that components of the matrix surface and volume elements are

$$
d X \wedge d X=\frac{\gamma_{\mu \nu}}{2 !}\left\{X^{\mu}, X^{\nu}\right\} d \sigma^{1} \wedge d \sigma^{2}
$$

for one forms $d x^{\mu}=\partial_{r} X^{\mu} d \sigma^{r}$ on the surface and

$$
d X \wedge d X \wedge d X=\frac{\gamma_{\mu \nu \rho}}{3 !}\left\{X^{\mu}, X^{\nu}, X^{\rho}\right\} d \sigma^{0} \wedge d \sigma^{1} \wedge d \sigma^{2}
$$

for one forms $d x^{\mu}=\partial_{\alpha} X^{\mu} d \sigma^{\alpha}$ on the world-volume. Therefore matrices $\mathcal{M}$ and $\mathcal{L}$ appear quite naturally when working with matrix line element $d X$.

Having in mind the above remarks let us consider the following analogy. In the case of point particles the Lagrangian formally can be written as a matrix

$$
\sqrt{\dot{x}_{\mu} \dot{x}^{\mu}} d \tau=\gamma_{\mu} \dot{x}^{\mu} d \tau=\gamma_{\mu} d x^{\mu}
$$

which has a counterpart in $-i \gamma^{\mu} \partial_{\mu}$ - the differential part of the Dirac operator. Likewise, for membranes we have

$$
\sqrt{G} d^{3} \sigma=\frac{i}{6} \gamma_{\mu \nu \rho}\left\{X^{\mu}, X^{\nu}, X^{\rho}\right\} d^{3} \sigma=i \gamma_{\mu \nu \rho} d \Omega^{\mu \nu \rho}
$$

where $d \Omega^{\mu \nu \rho}:=\frac{1}{6}\left\{X^{\mu}, X^{\nu}, X^{\rho}\right\} d^{3} \sigma$ is the element of the world-volume, hence the corresponding Dirac operator would be $\gamma^{\mu \nu \rho} \frac{\delta}{\delta \Omega^{\mu \nu \rho}}$, with the functional derivative (already considered by Nambu [13]). Therefore in the presence of the field $\mathcal{A}_{\mu \nu \rho}$ we would obtain

$$
\gamma^{\mu \nu \rho}\left(-i \frac{\delta}{\delta \Omega^{\mu \nu \rho}}+e \mathcal{A}_{\mu \nu \rho}\right) \Psi=0
$$

which is an alternative way to write the Dirac equation with the three-form.

\section{CONCLUSIONS}

A theory of extended objects aspiring to describe particles should include fermionic fields. How to write down such theory is non-trivial considering the fact that the starting point is a classical object described by bosonic fields (coordinates). A problem of this kind but for pointlike particles was solved by Dirac by taking a square root of the constraints and since it is the correct route we take it as a guiding principle in the attempt to include fermions into the theory of extended objects.

Because the constraints are local equations depending on the geometry of the membrane, the corresponding Dirac equation will be a functional one. Even for the simplest choice of the matrix $\mathcal{A}$, this results in a highly complicated system 10 which fortunately can be understood using the matrix regularization so that the masses 
of the membrane are hidden in the spectral properties of such matrix-Dirac operator. Still to find the eigenvalues of this equation is probably very difficult which is why we considered a semiclassical approach by concentrating on spherically symmetric membranes. The resulting system is a Dirac equation for the radius of the membrane. Although this approach is only approximate we believe the we have identified the crucial properties of the theory i.e. that the spectrum is discrete and consists of positive as well as negative eigenvalues - in particular there is no 0 eigenvalue and there are no tachyons. The negative masses are due to the negative tension and therefore such particles would be unstable.

Acknowledgments Discussions and the correspondence with J. Hoppe, M. Kuźniak and P. O. Mazur as well as the support from the Swedish Research Council, KTH and NORDITA are gratefully acknowledged.

* Electronic address: maciej.trzetrzelewski@gmail.com

\section{Appendix}

In order to obtain numerically the eigenvalues of matrix operator $H$ we use the cutoff method [15] which consists of calculating the representation of $H$ in some orthonormal basis (we choose $\frac{1}{\sqrt{(n+1)(n+2)}} x L_{n}^{(2)}(x) e^{-x / 2}$, where $L_{n}^{(2)}(x)$ are generalized Laguerre polynomials), truncate the infinite matrix (i.e. truncate each operator appearing in the entries of the $2 \times 2$ matrix $H$ ) and then numerically diagonalize it. The spectra and the eigenvectors of the truncated matrices converge to their exact counterparts. The results of the numerical approach are presented in Figure 1.

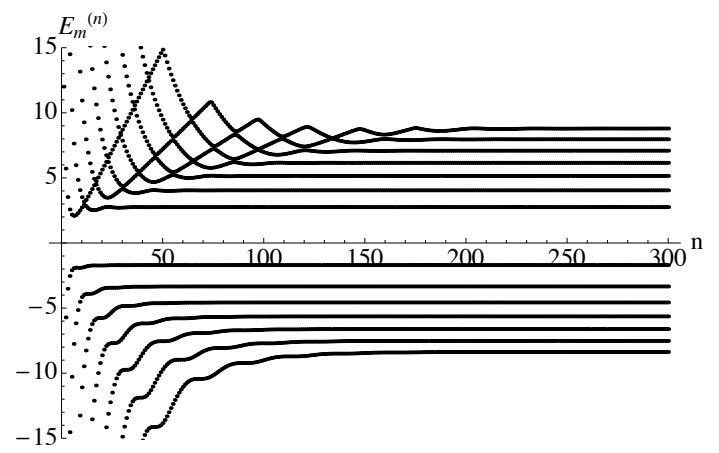

FIG. 1: Convergence of the energy levels with the cutoff up to $n=300$.

[1] P. A. M. Dirac, Proc. Roy. Soc. A268, (1962) 57-67; P. A. M. Dirac, Proceedings of a Conference held in Warsaw and Jablonna, July 1962, ed. L. Infeld, P. W. N. Publishers, 1964, Warsaw, 163-171; discussion 171-175; P. A. M. Dirac, Proc. Roy. Soc. A270, (1962) 354-356.

[2] M. J. Duff, Asim Barut Memorial Lecture, Bogazici University, Istanbul, October (2002).

[3] P. S. Howe, R. W. Tucker, J. Phys. A: Math. Gen. 10 L155 (1977); J. Hughes, J. Liu and J. Polchinski, Phys. Lett. 180B (1986) 370.

[4] E. Stedile, IJTP, Volume 43, Number 2, 385-397.

[5] A. O. Barut, M. Pavšič, Phys. Lett. B306 (1993) 49-54.

[6] C. Itzykson, J. B. Zuber, Quantum Field Theory, Dover Publications, Inc. Mineola, New York, 2005.

[7] P. Gnädig, Z. Kunszt, P. Hasenfratz, J. Kutti, Ann. of Phys., 1162 (1978) 380-407.

[8] J. Hoppe, Ph.D. Thesis MIT (1982).

[9] L. Smolin, Phys. Rev. D57 (1998) 6216-6223.

[10] V. Moncrief, Gen. Relativ. Gravit. (2006) 38(4) 561-575.

[11] J. Hoppe, PoS CNCFG2010 019 (2010).

[12] J. Hoppe, M. Trzetrzelewski, Nucl. Phys. B849 (2011) 628-635.

[13] M. Kalb, P. Ramond, Phys. Rev. D9 (1974) 2273; Y. Nambu, Phys. Rep. 23 (1976) 250; C. Teitelboim, Phys. Lett. B167 1 (1986) 63-68; M. Henneaux, C. Teitelboim, Foundations of Physics 16 (7) (1986) 593-617.

[14] Y. Nambu, Phys. Rev. D7 (1973) 2405-2412.

[15] M. Trzetrzelewski, Quantum mechanics in a cut Fock space, M.Sc. Thesis, Jagiellonian University, (2003). M. Trzetrzelewski, J. Wosiek, Acta Phys. Polon. B 35 (2004) 1615.

M. Trzetrzelewski, Acta Phys. Polon. B35 (2004) 2393. 